\title{
A review of immersivity in serious game with the purpose of learning media
}

\author{
Anang Kukuh Adisusilo ${ }^{1 *}$, Santirianingrum Soebandhi ${ }^{2}$ \\ ${ }^{1}$ Departement of Informatics Engineering, Faculty of Engineering, University of \\ Wijaya Kusuma Surabaya, Jl. Dukuh Kupang XXV/54, Dukuh Pakis, Indonesia \\ ${ }^{2}$ Department of Management, Faculty of economics and business, University of Wijaya \\ Kusuma Surabaya, Jl. Dukuh Kupang XXV/54, Dukuh Pakis, Indonesia
}

\begin{abstract}
Serious Games that have a specific purpose other than being an interesting or fun medium for users. In the concept of learning, it is as if you are giving the learning process to students who do not realize that the primary purpose of Serious Games is to learn. Various studies prioritize the immersive side of Serious Games to optimize their use so that players can take advantage of the main objectives of Serious Games. There is a difference in understanding between fun and reality, where Serious Games' main goal requires a data approach. However, if enter accurate data too advanced to create a real situation, it can detract from the fun side of a serious game. This review paper discusses various research results that prioritize immersion in designing and developing Serious Games, ranging from various methodologies to the factors that affect the success rate of Serious Games.
\end{abstract}

Keywords: Immersive, Serious game, Learning, Media.

\section{OPEN ACCESS}

Received: June 29, 2021

Accepted: July 20, 2021

Corresponding Author: Anang Kukuh Adisusilo anang65@uwks.ac.id

Copyright: The Author(s) This is an open access article distributed under the terms of the Creative Commons Attribution License (CC BY 4.0), which permits unrestricted distribution provided the original author and source are cited.

\section{Publisher:}

Chaoyang University of

Technology

ISSN: $1727-2394$ (Print)

ISSN: 1727-7841 (Online)

\section{INTRODUCTION}

Rapidly developing computer systems help fulfill human needs such as learning, marketing, entertainment, games, and even prediction. Humans find it easier to learn new knowledge in situations that are comfortable using technology (Ghavifekr and Rosdy, 2015; Whitaker, 2020). This comfortable situation can be created when there is a fun side of a system to understand and remember knowledge quickly. As for business people, the goal of a comfortable situation system is to make users comfortable in advertising and to make them more interested in the product so that more products are sold (Digitalcommons@uri and Jacobsen, 2017; Folkvord and van 't Riet, 2018; Kareem, 2021). User comfort for the system or a system giving the effect of feeling happy to the user can be realized in a game with a specific purpose in the system called a Serious Game.

Serious Game is a game used for self-pleasure in playing and as a medium for teaching, conveying information, and educating by combining elements of experimentation and emotional freedom by actively playing to provide the broadest stretch of mind to players (Abt, 1987). Serious Game is a broader definition of "game" because every part of the device that is made is not only intended for entertainment, but there is a particular purpose with its structure of a game (Sawyer and Smith, 2008). Serious gaming is not just to make users happy. Still, there are elements of imagination and provide knowledge and games used pedagogically for political, social, marketing, economic, environmental, or other purposes (Ma et al., 2011).

Some researchers distinguish between Serious Game and games for learning. In general, whatever form of the Game is used for Education is serious play. Even so, it can still be categorized as a Serious Game if the main goal is more than the fun side. Most game designers and researchers agree edutainment game is a serious part of gaming 


\section{International Journal of Applied Science and Engineering}

Adisusilo et al., International Journal of Applied Science and Engineering, 18(5), 2021229

(Sawyer and Smith, 2008; Calderón et al., 2017; De Gloria et al., 2014; Droso s et al., 2018; Nima et al., 2019; Rankin et al., 2008; Ratan and Ritterfeld, 2009; Vlachopoulos and Makri, 2017).

\section{SERIOUS GAME IN EDUCATION}

The main objective of Education is the delivery of textbook knowledge, and there is a combination of entertainment and Education to increase student motivation or strengthen the learning paradigm; edutainment is a game that is a Serious Game (Shen et al., 2009). Another concept that is very much alike and partially overlaps with the Serious Game category is e-learning and digital-based learning. The concept of e-learning has become popular as serious gaming, moreover, it has also become the topic of research in various disciplines, among others are psychology, pedagogy, or computer science and information technology (Haythornthwaite et al., 2016). It is as much the same as entertainment education terms, the conception of elearning refers to any computer-based learning. Unlike entertainment education that is not attached to any particular medium, e-learning is integrated with computers.. Elearning does not imply a need for entertainment and pleasure in the learning process. The main advantage of elearning is that it allows distance learning and learners to be distributed in different locations and non-parallel or asynchronous and flexible learning. E-learning is basically about the flexibility of learning in time and space. Using the broadest possible definition of e-learning as computerbased learning of any kind, one can include serious gaming as a subcategory (Koubek and Macleod, 2004; Pivec and Kearney, 2007).

The serious forms of digital gaming are parts of elearning, yet, the terms of serious games still overlap with another term, such as game-based learning (GBL) or digital game-based learning (DGBL) (Prensky, 2007). However, those terms are slightly different. Game-based learning is more likely refers to the pre-video game definition of serious gaming. While digital game-based learning (DGBL) is identical to the modern use of 'Serious Games' for computer video games with/for educational purposes. However, the initial definition of DGBL was more in line with the characteristics of e-learning as he dubbed DGBL "any learning on the computer or online" (Prensky, 2007). Fig 1 describes the relationship between edutainment, Game-based learning (GBL), Digital Game Base learning (DGBL), e-learning, and Serious Games.

From some opinions, in a Serious Game, fun elements can still be provided regardless of the main goal. So that by using Serious Games, you can achieve your goals while still making users happy in the classification of Serious Games. As in educational training and games, they are increasing fun generates interest and positive emotions by using ludative learning methods (Sanchez, 2013). Research has been carried out on the implementation of games for training and learning, such as training on tillage with a plow (Adisusilo et al., 2018; Adisusilo et al., 2020), educational facilities in the sport's economics undergraduate program, namely the Serious Game Top Eleven (Afthinos et al., 2021). There are also Serious Games whose aim is to make students play an active role in the learning process and is as tool to gain knowledge so that playing is more adaptive and effective (Arnold et al., 2013; Wassila and Tahar, 2012).

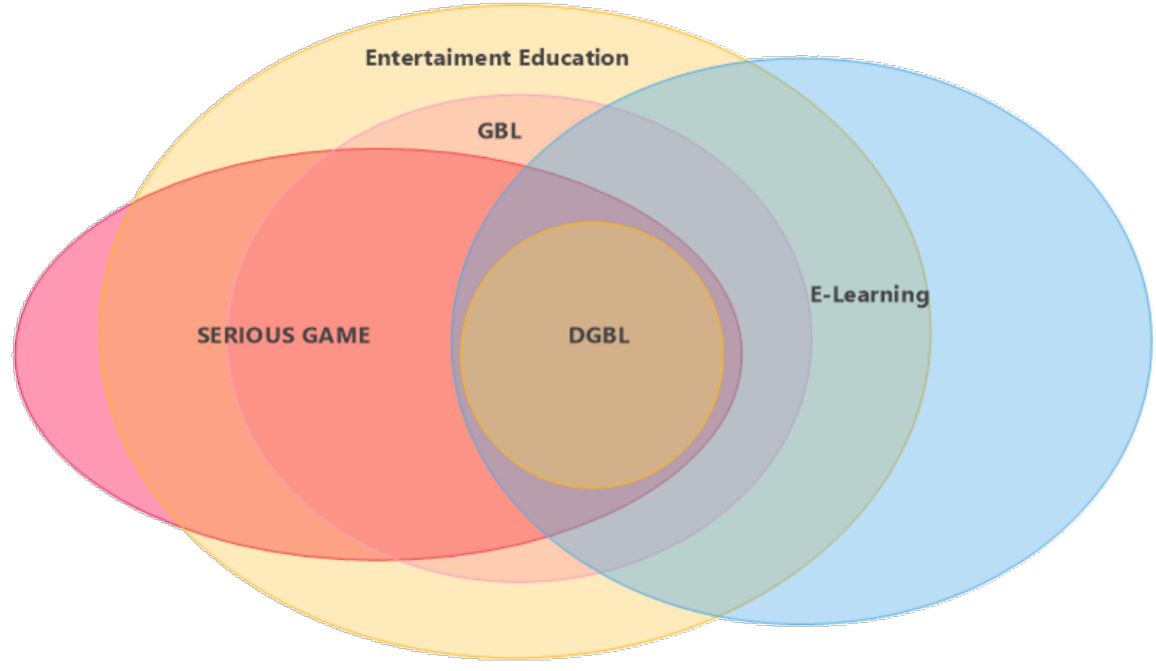

Fig 1. The relationship between Edutainment, Game Base learning (GBL), Digital Game Base learning (DGBL), Elearning, and Serious Games 


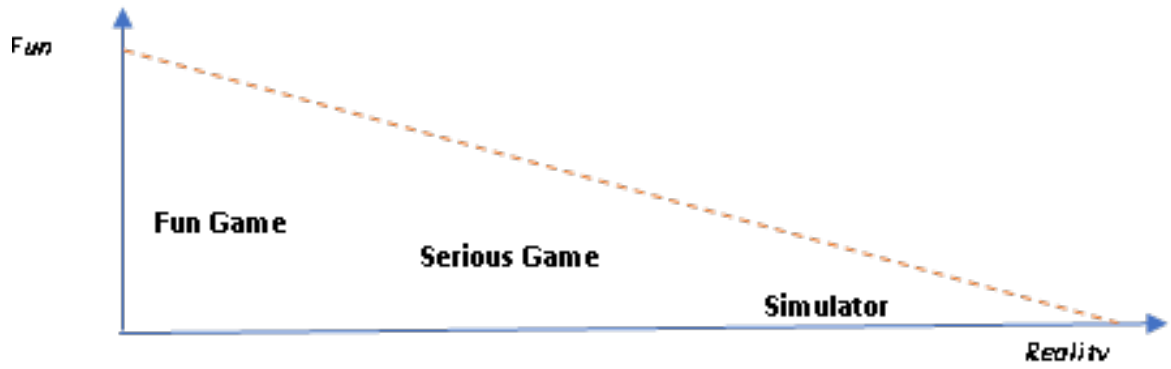

Fig. 2. Fun game, Serious Game, and Simulator correlation diagram from fun and reality point of view

From the various explanations, while there is still a fun side to the game, one of the Serious Games is suitable for learning media rather than just a fun game. From another perspective, Is the Serious Game and simulator are the same? Then from the point of view of trying to provide actual data in a system, a Serious Game and simulator are almost similar, such as a commonly used mathematical process simulator such as Matlab - Simulink (commercial) or Scilab - Scicos (freeware). There is no flow for fun in the system simulator that makes users even more interested in using it, although it is still possible to have a fun side with a Serious Game concept. Such as simulators for heating operations used in the hydrocarbon and chemical processing industry (HPI / CPI) to heat liquid hydrocarbons converted to fuels such as gasoline or chemicals such as ethylene, the aim of providing realistic training before they work with actual equipment (Baukal and Bussman, 2009).

Another example is the simulation of building a PC-based flight test laboratory experiment to detail the flow of experiments carried out (Smith, 2001). There can also be research to analyze the impact of "correcting feedback" (i.e., feedback showing only student errors) and "reflective feedback" (i.e., feedback including clues to encourage students to rethink specific answers) on student learning effectiveness (Jaeger and Adair, 2019). From some of these examples, the data approach is used to achieve reality. Still, there is no mention of the side that makes simulator users interested in using the simulator if it has not been successful.

Whereas the fun game method where, in a game that only aims to have fun, the effects caused mainly on players are eudaimonic and hedonic. As in the article on digital game exploration, it is argued that adolescents cite narrative aspects more (i.e., a game story, characters, moral choices). The players to close for real-world players needs audiovisual elements such as graphics and soundtracks as important triggers of the eudaimonic playing experience (Daneels et al., 2020).

Whereas the article on the potential of CA for service firms by creating social closeness and enabling fast and measured communication with customers suggests a game that can make a social presence an essential predictor of hedonic and utilitarian value and corporate goals in business (Bedué, 2020). In an article examining the effectiveness of games and films in student learning to revive courses, games could bring fun and pop culture to international relations classes, compared to using films (Brandle, 2020). The relationship between Fun Game, Serious Game, Simulation, shown in Fig. 2.

\section{EDUCATION GAME IN SERIOUS GAME CLASSIFICATION}

The concept of a Serious Game with a particular purpose other than the fun side is for learning/training purposes, so it is often called the Edu Game. The design of the Serious Game model aims to feel that playing the game will get the knowledge gained from the consequences when playing (Barbosa et al., 2014) so that Serious Games are not boring. In basic game design, the main domain is a pedagogy that prioritizes design for learning purposes, games that prioritize game design that is not boring. Fidelity prioritizes simulation designs that are the same as reality (Rooney, 2012). Regardless of the purpose for learning, the Serious Game can be classified based on the criteria and dimensions of "Serious and Game."(Sawyer and Smith, 2008).

Criteria Serious Game

According to Serious Game taxonomy, these criteria are divided into single and multiple criteria (Sawyer and Smith, 2008). These recommendations can be split based on "market-based" and "goal-based.". in single market-based criteria are Healthcare games, Public policy games, Strategic Communication games, Defense games, and Training and Education games (Zyda, 2005). Then, research (Michael, 2006) includes Military Games, Government Games, Cooperate games, Healthcare Games, Political Games, Religious Games, and Art Games. In addition, according to Alvarez and Michaud (2008), several games are classified as single criteria market-based, namely Advertising Games, Information \& Communication Games, Culture Games, Activism Games. In this opinion, edugame (game based on education), including training, is stated together to be categorized as a single market-based criterion of Serious Games. The single purposed established criteria according to (Saurina et al., 2016; Bergeron, 2006; Bogost, 2005), Edugame is part of these criteria (De Gloria et al., 2014; Nima et al., 2019).

Classification Serious Game base dimension "serious" and "game."

Based on the concept of Serious Games purpose, almost all researchers agree that Edu games fall into this criterion 
(Calderón et al., 2017; Drosos et al., 2018; Vlachopoulos and Makri, 2017). The Serious Games with their concept of combining five components consisting of Rules, Input methods, Spacer-related setup, Time-related setup, Dramarelated is apart from their purpose and scope of the game series itself. Furthermore, Serious games basically can be distinguished based on its concept of learning in educational games, namely Information Games in News, Persuasive Political Games and Advertising Games, Subjectively Military Games and Art Game, and based on the coverage of the Serious Games, namely topic/market and audience/public user (Sanford et al., 2015; Sanmugan et al., 2014; Valladares-Rodríguez et al., 2016).

\section{IMMERSIVITY IN SERIOUS GAME}

A game that can absorb the player into the game world then consistently feels in it. "Suspension of Disbelief" or a mental state that the player reaches when the player is completely immersed in forgets that he is playing the games (Iii and Ogden, 2005). The concept of fidelity supports this situation, or it can cause players to be loyal or to continue to follow all the gameplay in the Serious Game. The concept of fidelity in the Serious Games points out to the extents to which the games imitate the natural world. Alexander et al. (2005). Two levels of fidelity known in the Serious Games which are physical fidelity and functional fidelity. The first is refers to the degree of the game environment looks, sounds, and feels natural accordingly, physical fidelity is determined by factors including visual appearance, control, audio, and variable physical model of player control (Maran and Glavin, 2003).

The latter refers to the extent the game environment simulate the real world response to the player actions including game narrative and interactivity elements. Alexander et al. (2005). The rationale for fidelity in the Serious Game comes from a dual pedagogical goal: (a) exciting and immersive and (b) presenting a practical learning experience. Besides being supported by a realistic and $3 \mathrm{D}$ visual display, from the perspective of experiential learning, fidelity is the assurance that the results are following the actual situation to evoke the player's experience as in the real world (Rooney, 2012). Three levels relate to how players feel when they are playing and affect the immersiveness of the games (Brown and Cairns, 2004; Zemliansky and Wilcox, 2010; Brown et al., 2004), namely Engagement, Engrossment and Total Immersion.

\subsection{Engagement}

Access to playing games is a player's initial/basic involvement with the game, which is a preference that can easily remove barriers that hinder interest in playing. For example, with easy controls, even just tinkering with the buttons, a game can run, and the player will become proficient on their own. Time investment into the game, in playing it will require a lot of time and energy, so the barriers that make players feel that they are wasting time playing the game must be overcome. The player's effort to reach the target game requires much energy in playing. So we need a reward, usually in the form of a score or game bonus, to give players satisfaction in playing. Removing barriers to uninteresting initial preferences makes players feel like they are not wasting time and energy playing. The players involved will be interested in the game and want to continue playing.

Immersivity is a graded involvement (Brown and Cairns, 2004). The concept of engagement in the Serious Game with the level of participation of the players has been carried out in several recent studies. With a simple video game scenario plot to train health workers in maintaining hand hygiene to prevent the transmission of pathogens and infections related to health care, it is claimed to function as an educational medium (Sax and Longtin, 2011). This is associated with the concept of Immersion with a repeated and straightforward scenario that can be listened to players to provide education indirectly to increase user engagement. In the Immersive Serious Game research, which aims to train electricians in critical activities, namely routine maintenance, the electrical power station includes an NPC (non-player character) to be more adaptive in making decisions while the maintenance process is in progress. The obstacle in the game is more likely to be able to increase user experience; this is a User Experience (UX) concept that can add to user engagement (de Geus et al., 2020). Optimization calculations from the depth of the agricultural land preparation process are based on actual conditions to help feedback information in the gameplay scenario. It is expected that user experience will arise in the game by increasing the Serious Game's immersive side (Adisusilo et al., 2018; Adisusilo et al., 2020).

Another research is immersive virtual reality that virtual Immersion can be used to develop collaborative educational applications. Multiple users can collaborate in a virtual common room and communicate with each other via voice. With the collaborative environment for geography education, case studies are developed and evaluated. The experimental visualization scenario explores the possibility of studying geography in a collaborative virtual environment. (Doležal et al., 2017). In doctrinal training, the army used immersive game-based in peak training instruction, incorporated into real-time strategy games intended to train and assess their understanding and compliance (Bond et al., 2017). In an experiment using original content from Europe's top orchestras to improve the learning aspects of listening to music, for education and personal enrichment, audio signal processing was used to separate different sound sources (instruments) in an acoustic (orchestral) (Janer et al., 2016). This research shows that achieving immersiveness in a system scenario in the form of system flow, audio, video, or algorithm optimization increases engagement. 


\subsection{Engrossment}

The excitement in the game is greatly influenced by design in making the game so that players, post-playing, feel drained of energy because it is a game that is appealing and cool to play so that player does not feel drained of energy when the games is finished. Some of the things that are used to plan this game are the details of the visual design of the game to make it look luxurious, interesting tasks or targets in the game based on scenarios from the game story, as well as exciting plots or games as well as plans in the game environment, thus making players curious.

Based on the design-making of the game, players can feel drained of energy because it is fun when playing. This is an engrossment concept. Some researches has also been done. Such as for the introduction of history or early culture to the younger generation can be taught using immersive virtual applications: using gamification technology, technological and economical accessibility of the VR HMD (headmounted display) viewers. The use of this technology is desirable and increases the Engrossment of users (Luigini and Basso, 2021). Another cultural research within the framework of a case study of cultural heritage, by applying part of a Serious Game called Hippocratica Civitas Game, in which the gameplay comes from the historical sites of San Pietro a Corte and Palazzo Fruscione in the city of Salerno, Italy, and to solve puzzles. The results of the game evaluation show that the game is effective in terms of: firstly, gaining knowledge, secondly, participants who enjoy the game are deeply involved in profound experiences, and finally, assess the game positively for educational learning in cultural heritage (Andreoli et al., 2017). The research that puts forward scenarios in the narrative to form Serious Games to teach certain cultures effectively supported by several narratives, educational, immersion theories, educational games are culturally relevant and immersive (Baker et al., 2017).

In another cultural study, I-Ulysses research, a virtual reality game designed to be based on the famous work of Ulysses by Irish writer James Joyce. Aspects of gamification are explored at I-Ulysses through locks, including focusing on virtual worlds and crowd intelligence based on real-world data to determine how Serious Games can be used in these cultural heritage preservations and knowledge transfer principles. With the concept of immersive virtual and gamification to interact with IUlysses, the Serious Game can see that the application presented through a virtual reality lens provides an informative and educational guide for Ulysses to engage and expand the audience (O'Connor et al., 2020). The framework of designing learning games to provide students with a more immersive gaming experience was proposed in the research to create a gaming environment combined with arcade games and serious learning games to provide students with a deeper and better gaming experience. (Lv et al., 2018; Yang et al., 2019). By adding the Oculus Rift device to increase immersivity accompanied by user activity, the engagement side will be higher in-game education (Loup et al., 2016).

Learning technology with the immersive Serious Game can be applied to the development of UI and UX for the concept of Heuristic Evaluation and Mobile Instruction (Gordon et al., 2016). Besides that, the application of HMD (helmet-mounted display) to educate passengers about flight safety has successfully shown that serious deep play is more exciting and evocative fear rather than security cards (Chittaro and Buttussi, 2015). Based on game-based creative learning as a combination of constructive, immersive, and reflective aspects, research is carried out using "games" in mathematics education. The game can use that mathematical thinking to think about game scenarios as a whole, to regulate the competitive aspects of the game, and ensure that the gameplay is fair and balanced. for all users (Misfeldt and Gjedde, 2015). The DoCENT (Digital Creativity ENhanced in Teacher education) project cofunded by the EU's Erasmus + program aims to enhance digital creativity in the context of Early Training Education (ITE). The study describes a DoCENT game prototype and discusses a model that includes five steps. In particular, focus on the co-creation process to design learning scenarios with actual Serious Game teachers and users. In addition, this paper shows the learning scenario methodology, the interactions of the players, and the feedback obtained from the adaptive tutoring system (Di Fuccio et al., 2020). From various studies, the combination of tools, UI, UX, and scenarios that support more user interaction is designed to increase attractiveness and engagement, namely Engrossment, because the fun of playing is a concept of user preoccupation in games, thus increasing Serious Game immersion.

\subsection{Total Immersion}

In this section, the game must make the player get into the game's atmosphere so that the experience arises for the player. The limitation of total Immersion is that it can not include player empathy or attachment to the game's atmosphere. Player empathy is usually tied to the characters of the game. At the same time, the atmosphere is the environment of the game in the game. Therefore, combining various elements such as graphics, sound, and gameplay (scenarios and stories) is crucial. So that in making games with total Immersion, what needs to be considered are the visual, auditory, and mental elements of the players.

In Total Immersion, users feel the real situation so that the Virtual Reality model, Virtual environment, is integrated into Serious Games. Total Immersion can be realized with accurate data for Serious Game system processes manifested in Serious Gameplay and game scenarios. The use of camera technology to control the game so that the player seems to be monitoring / holding the agricultural equipment machine used in the form of Serious Games for agricultural training because if using traditional training requires high training costs, low efficiency, and the risk of 
accidents (Wang et al., 2013). The development of a Serious Game to achieve total Immersion requires equipment of control and sensors. Besides combining multimedia elements and adaptive gameplay flow, such as using the Immersive virtual reality concept, it can make users feel like they are in real life. Research in emergency medicine education and training uses the Serious Game of VR has been successfully assessed as a practical educational approach and increases training effectiveness in enhancing through "presence experiences" or reducing the perceived limitations of using virtual media (Lerner et al., 2020).

Another research is to develop hardware and software to enhance Serious Game immersion to achieve total Immersion. Included in professional training media is about how to use interactive models to enhance medical imaging and machine operational safety training in technical facilities and laboratories. In the form of an information model in a simulation, machine functional safety training can develop a realistic virtual environment (Bernal, 2020). In addition to the user control side, sound must improve in a Serious Game. The multimedia side, including audio, must also be enhanced to increase immersiveness. By creating a convincing environment that must be supported by audio, which is known as environmental modeling, that is closer to reality, audio is important in such immersiveness (Sinclair, 2020).

The concept of immersiveness is realized by combining virtual reality and Serious Games with interactive and attractive models. For example, it is useful and effective for self-rescue exercises during an earthquake for children. More adaptive gameplay is developed so that pedagogical strategies use different contexts and content. (Feng et al., 2020, Feng et al., 2021). In research with the CREAM model for Civic Education, Historical Relevance, Engagement, Applicability, and Multimodality, which aims to introduce archaeological and historical content into the classroom in the form of the IVR Carthago Nova video game. Immersive virtual reality (IVR) is needed in the category of Total Immersion to make the goal work better (Egea-Vivancos and Arias-Ferrer, 2020). Cognitive and motor skills are the case for Percutaneous Coronary Intervention (PCI), a standard procedure in which a hemodynamic professional removes narrowing or blockages of the coronary arteries trained using Immersive VR and concludes. That it has potential as a learning tool for medical education using VR technology.

VR is part of the Serious Game because it still prioritizes certain goals and has a fun side for its users (Perez-Gutierrez et al., 2020). In addition, the research combines five aspects of STEAM education (a learning approach that integrates Science, Technology, Engineering, Arts, and Mathematics as a pathway to guide student inquiry, discussion, and critical thinking.) Which is combined with the ARCS motivation model (group motivation theory: attention (A), relevance $(\mathrm{R})$, trust $(\mathrm{C})$, and satisfaction $(\mathrm{S})$.) The research shows that an Immersive VR (IVR) learning mechanism can effectively trigger students' motivation to learn new knowledge while enhancing learning (Wang et al., 2018). Virtual Reality (VR) and Virtual Environment (VE) equipment - then Oculus Rift, Microsoft Hololens, and Sony Playstation VR - is a solution to provide users with an immersive interactive experience in the concept of off-theshelf technology into Augmented Learning. Become the primary realistic tool. Most systems are built with a game model using a game machine, where a game with a particular purpose that is a Serious Game can have a learning effect as the desired consequence of playing (Gordon and Brayshaw, 2017).

As a groundbreaking form for law students, even the Westminster University Law and Computer Science department can explore real case scenarios using Virtual Reality (VR) technology to discover key pieces. Evidence from real scenarios and decide whether a criminal case is a murder or not. Called REVRLaw (REal and Virtual Reality Law), REVRLaw integrates Immersion into VR as the perception of being physically present in the non-physical world. In the Serious Game, the prototype and mechanism are used to focus on the criminal case and make the best use of this immersive learning approach (Mentzelopoulos et al., 2016). Interactive technology based on the user's movements and gestures in physical space is overgrowing, widely used in entertainment games, such as Kinect-based games. Teknologi interactive can incorporate the ability to explore outer space through Kinect-based games into Serious Game design. Therefore, as an example, Kinect applied the ancient world map, Kunyu Quantum, from the National Palace Museum collection in Serious Game development (Peng et al., 2015). The Oculus Rift DK2 is used in experiments to provide immersive virtual reality with affective scenarios for application fields such as health, education, and training.

Designing interactive games that are responsive to user emotions increases user effectiveness and acceptance. A major factor in user interactions is emotional reactions to game scenarios, which has motivated us to analyze immersive games using biofeedback ( $\mathrm{Li}$ et al., 2015). Perceptual input is an area that appears in HCI which suggests that the Development of a Perceptual User Interface (PUI) is a part that encourages total Immersion so that Serious Game users using cellular can form an immersive social networking environment because there are various kinds of input devices, software platforms, interaction possibilities, and various ways to combine all of the above elements in achieving the PUI concept (Mentzelopoulos et al., 2015). VR-based fire safety training for healthcare staff at Vincent Van Gogh Hospital (VVG) in Belgium demonstrates that VR SG is more effective than slide-based lectures in terms of knowledge acquisition and retention and increasing self-efficacy in the short and long term than college; slide-based (Rahouti et al., 2021). 


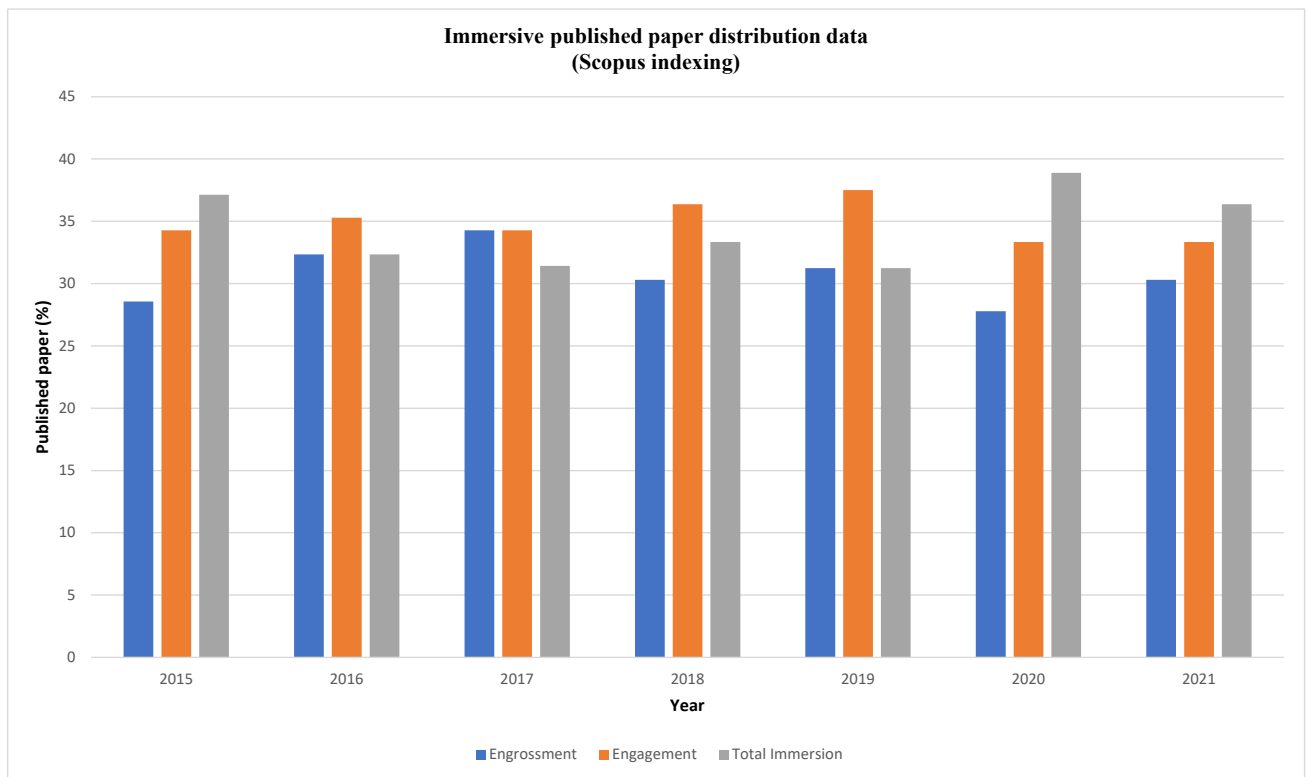

Fig. 3. Immersive data distribution chart of published papers (Scopus indexing)

\section{DISCUSSION AND SUMMARY}

This article uses Harzing's Publish and Perish's tools using keywords; immersive, immersive Serious Games, and immersive educational games. By searching the two groups of papers indexed by Scopus and Crossref in the past six years, we found 107 articles indexed by Scopus and 200 papers indexed by Crossref. The 200 paper indexed Crossref contained the word immersive in the paper title, 172 or $86 \%$ discussed immersivity. In contrast, of the 107 Scopus papers, only 25 included the word immersive in the title, or only $23 \%$ focused on immersivity. So the percentage can interpret that research on Immersion in Serious Games is interesting research. Still, many factors influence or involve many disciplines to do this research, so the trend of immersive research is less than the internal game itself. The literature review focused on Immersion as the main objective of the study. Literature took most of the cases from Scopus immersive indexing, which according to research trends in the last six years. It was grouped according to three levels of Immersion, between research with the concept of development towards total Immersion and balanced Engrossment. At the same time, those that increased engagement were less, as shown in the graph in Fig. 3.

From the three levels of Immersion, namely engagement, Engrossment, and Total Immersion, researchers always try to get to total Immersion, but many factors influence total Immersion. For example, Serious Game developments that continue until now, especially in the concept of immersiveness, are; Microsoft Flight Simulator, which has been around for 39 years, has been developing a serious gaming concept since 1982 with the release of Microsoft Flight Simulator 1.0., The gameplay is still a single player with limited gameplay rules, namely: The starting airport is Meigs Field in Chicago, with views of the city skyline to the left and Lake Michigan to the right. This will remain the default airport in future versions of Microsoft Flight Simulator until the actual airport is closed and RGB display is limited to the IBM PC, a Serious Game concept as a training medium for aspiring airplane pilots, for aspiring pilots to practice piloting an airplane (Hockman, 1987). Simple gameplay can already reflect the actual situation of the airport because this flight simulator system will be completed in one gameplay rule when the airport closes. This gameplay is part of the concept of presenting actual data to users, in this case, the Microsoft flight simulator version. This makes the player feel can dive deeper into the game and participate in the reality of airplane flights at the airport. Besides that, the dashboard display that has been made follows reality-airplane steering button condition.

The concept of engagement is still emphasized in this version, as the development of version 1 appeared in 1984 with the addition of joystick and mouse input, as well as support for RGB monitors (4-color CGA graphics), the IBM $\mathrm{PC}$, and (in later versions) ) Hercules graphics and LCDs for laptops. The new simulator expanded the scenery coverage to include a model of the entire United States, although the airports were limited to the same areas as in Microsoft Flight Simulator 1.0. This version also puts forward the concept of engagement because the graphic supporters have not yet reached reality (PC Mag 1984-10-02, 1984; PC Word, 1984). The idea of engineering has been used following Windows 95, namely Microsoft Flight Simulator 1995, with more scenes and scenarios in gameplay and more aircraft. Notice the texture mapped runway, planes, and sky.

The high density of 3-D buildings continues to increase in the Engrossment concept to players until 2000, including aircraft type according to conditions at the airport. This version was also released with support for Microsoft Sidewinder Joystick Pro Force Feedback, allowing players 
to receive multiple sensory inputs from simulated trim forces on airplane controls (Moore, 2007). Furthermore, it continues to be developed until now so that it can achieve total Immersion with various supports such as weather simulations based on actual weather conditions, flight schedules according to natural, and also the number of luggage and real passengers (Simulator, 2019; Simulator, 2020)

The development of a Serious Game is carried out in stages by prioritizing the concept of continuous immersiveness. Accordingly, the need for actual data and actual conditions to form the gameplay side, control, and support for environmental modeling are also needed in the form of images, videos and even audio. The imbalance between gameplay, user control, photos, video, and audio causes the immersive concept to have not reached total Immersion or is still at the level of engagement and Engrossment or partial Immersion. With total Immersion, the specific goal of a Serious Game that is not just entertainment can be adequately achieved, especially in terms of learning material conveyed through games can lead to a good user experience so that it is effective in learning.

\section{REFERENCES}

Abt, C.C. 1987. Serious games. University Press of America. Adisusilo, A.K., Hariadi, M., Yuniarno, E.M., Purwantana, B. 2020. Optimizing player engagement in an immersive serious game for soil tillage base on Pareto optimal strategies. Heliyon, 6, e03613. https://doi.org/10.1016/j.heliyon.2020.e03613

Adisusilo, A.K., Hariadi, M., Yuniarno, E.M., Purwantana, B., Radi, R., Radi, R. 2018. Soil porosity modelling for immersive serious game based on vertical angle, depth, and speed of tillage. International Journal of Advances in Intelligent Informatics, 4, 107. https://doi.org/10.26555/ijain.v4i2.215

Afthinos, I., Manasis, V., Chrysanthopoulos, T.-P. 2021. Serious game top eleven as an educational tool in sports economics. International Journal of Serious Games, 8, 319. https://doi.org/10.17083/ijsg.v8i2.420

Alexander, A., Brunyé, T., Sidman, J., Weil, S.A. 2005. From gaming to training: A review of studies on fidelity, immersion, presence, and buy-in and their effects on transfer in pc-based simulations and games. DARWARS Training Impact Group, November, 14. https://doi.org/10.1016/j.athoracsur.2004.02.012

Alvarez, J., Michaud, L. 2008. Serious Games: Advergaming, edugaming, training and more. In IDATE 2008 BP 4167, France. http://www.ludoscience.com/EN/diffusion/285-SeriousGames-Advergaming-edugaming-training-andmore.html

Andreoli, R., Corolla, A., Faggiano, A., Malandrino, D., Pirozzi, D., Ranaldi, M., Santangelo, G., Scarano, V. 2017. A framework to design, develop, and evaluate immersive and collaborative Serious Games in cultural heritage. Journal on Computing and Cultural Heritage, 11. https://doi.org/10.1145/3064644

Baker, J., Wanick, V., Asiri, M., Wills, G., Ranchhod, A. 2017. Immersion and narrative design in educational games across cultures. In Serious Games and Edutainment Applications: Volume II (605-621). Springer International Publishing. https://doi.org/10.1007/978-3-319-51645-5_26

Barbosa, A.F.S., Pereira, P.N.M., Dias, J.A.F.F., Silva, F.G.M. 2014. A new methodology of design and development of Serious Games. International Journal of Computer Games Technology, 1-8. https://doi.org/ $10.1155 / 2014 / 817167$

Baukal, C., Bussman, W. 2009. Simulator for teaching process heater operating principles. ASEE Annual Conference and Exposition, Conference Proceedings. https://doi.org/10.18260/1-2-5290

Bedué, P. 2020. Just fun and games? utilitarian and hedonic chatbot perceptions and their role for continuance intentions. Lecture Notes in Business Information Processing, 389 LNBIP, 291-306. https://doi.org/10.1007/978-3-030-53337-3_22

Bergeron, B.P. 2006. Developing Serious Games. Hingham Mass by Charles River Media.

Bernal, I.F.M. 2020. System for immersive medical and engineering training based on Serious Games. Ingenieria y Universidad, 24. https://doi.org/10.11144/ javeriana.iued24.sime

Bogost, I. 2005. Frame and metaphor in political games.

Bond, A.J.H., Brimstin, J., Carpenter, A. 2017. Immersive games and expert-novice differences. Advances in Intelligent Systems and Computing, 498, 699-711. https://doi.org/10.1007/978-3-319-42070-7_65

Brandle, S.M. 2020. Games, movies, and zombies: Making IR fun for everyone. Journal of Political Science Education, 16, 459-478. https://doi.org/10.1080/ 15512169.2019.1568880

Calderón, A., Ruiz, M., Orta, E. 2017. Integrating Serious Games as learning resources in a software project management course: The case of ProDec. Proceedings2017 IEEE/ACM 1st International Workshop on Software Engineering Curricula for Millennials, SECM, 21-27. https://doi.org/10.1109/SECM.2017.3

Chittaro, L., Buttussi, F. 2015. Assessing knowledge retention of an immersive serious game vs. a traditional education method in aviation safety. IEEE Transactions on Visualization and Computer Graphics, 21, 529-538. https://doi.org/10.1109/TVCG.2015.2391853

Daneels, R., Vandebosch, H., Walrave, M. 2020. Just for fun?: An exploration of digital games' potential for eudaimonic media experiences among Flemish adolescents. Journal of Children and Media, 14, 285-301. https://doi.org/10.1080/17482798.2020.1727934

de Geus, K., Beê, R., Corrêa, V., Santos, R., Faria, A., Sato, E., Swinka-Filho, V., Miquelin, A., Scheer, S., Siqueira, P., Godoi, W., Rosendo, M., Gruber, Y. 2020. Immersive 
Adisusilo et al., International Journal of Applied Science and Engineering, 18(5), 2021229

serious game-style virtual environment for training in electrical live line maintenance activities. Proceedings of the 12th International Conference on Computer Supported Education, 2, 42-53. https://doi.org/10.5220/0009343200420053

De Gloria, A., Bellotti, F., Berta, R. 2014. Serious Games for education and training. International Journal of Serious Games, 1. https://doi.org/10.17083/ijsg.v1i1.11

Di Fuccio, R., Ferrara, F., Di Ferdinando, A. 2020. The DoCENT game: An immersive role-playing game for the enhancement of digital-creativity. Advances in Intelligent Systems and Computing, 1008, 96-102. https://doi.org/10.1007/978-3-030-23884-1_13

Doležal, M., Chmelik, J., Liarokapis, F. 2017. Ân immersive virtual environment for collaborative geovisualization. 2017 9th International Conference on Virtual Worlds and Games for Serious Applications, VS-Games 2017 Proceedings, 272-275. https://doi.org/10.1109/VSGAMES.2017.8056613

Drosos, V., Alexandri, A., Tsolis, D., Alexakos, C. 2018. A 3D serious game for cultural education. 2017 8th International Conference on Information, Intelligence, Systems and Applications, IISA 2017, 2018-January, 1-5. https://doi.org/10.1109/IISA.2017.8316442

Egea-Vivancos, A., Arias-Ferrer, L. 2020. Principles for the design of a history and heritage game based on the evaluation of immersive virtual reality video games. ELearning and Digital Media. https://doi.org/10.1177/2042753020980103

Feng, Z., González, V.A., Mutch, C., Amor, R., CabreraGuerrero, G. 2021. Instructional mechanisms in immersive virtual reality Serious Games: Earthquake emergency training for children. Journal of Computer Assisted Learning, 37, 542-556. https://doi.org/10.1111/jcal.12507

Feng, Z., González, V.A., Mutch, C., Amor, R., Rahouti, A., Baghouz, A., Li, N., Cabrera-Guerrero, G. 2020. Towards a customizable immersive virtual reality serious game for earthquake emergency training. Advanced Engineering Informatics, 46. https://doi.org/10.1016/j.aei.2020. 101134

Gordon, N., Brayshaw, M. 2017. Flexible virtual environments: Gamifying immersive learning. Communications in Computer and Information Science, 714, 115-121. https://doi.org/10.1007/978-3-319-58753$0 \_18$

Gordon, N., Brayshaw, M., Aljaber, T. 2016. Heuristic evaluation for serious immersive games and $M$ instruction. Lecture Notes in Computer Science (Including Subseries Lecture Notes in Artificial Intelligence and Lecture Notes in Bioinformatics), 9753, 310-319. https://doi.org/10.1007/978-3-319-39483-1_29

Haythornthwaite, C., Andrews, R., Fransman, J., Meyers, E. M. (Eds.). 2016. The SAGE Handbook of E-learning Research (SECOND EDITION). SAGE Publications Inc. Hockman, D. 1987. Bruce Artwick's flight simulator / you've come a long way, baby! / The History of an Epic
Program. Computer Gaming World, 36, 32-34. http://www.cgwmuseum.org/galleries/index.php?year=1 $987 \& \mathrm{pub}=2 \& \mathrm{id}=36$

Iii, R., Ogden, S. 2005. Game design: Theory \& practice. In Design. https:/gamifique.files.wordpress.com/2011/11/ 5-game-design-theory-and-practice.pdf

Jaeger, M., Adair, D. 2019. Process teaching simulator: Trial and error, thinking, learning effectiveness. IEEE Global Engineering Education Conference, EDUCON, April2019, 160-165. https://doi.org/10.1109/EDUCON.2019. 725190

Janer, J., Gómez, E., Martorell, A., Miron, M., De Wit, B. 2016. Immersive orchestras: Audio processing for orchestral music VR content. 2016 8th International Conference on Games and Virtual Worlds for Serious Applications, $\quad 2016$. https://doi.org/10.1109/VS-GAMES.2016.7590352

Koubek, A., Macleod, H. 2004. Game based learning (1519). PABST Science Publishing. https://www.research. ed.ac.uk/en/publications/game-based-learning

Lerner, D., Mohr, S., Schild, J., Göring, M., Luiz, T. 2020. An immersive multi-user virtual reality for emergency simulation training: Usability study. JMIR Serious Games, 8, e18822. https://doi.org/10.2196/18822

Li, Y., Elmaghraby, A.S., Sokhadze, E.M. 2015. Designing immersive affective environments with biofeedback. Proceedings of CGAMES 2015 USA - 20th International Conference on Computer Games: AI, Animation, Mobile, Interactive Multimedia, Educational and Serious Games, 73-77. https://doi.org/10.1109/CGames.2015.7272965

Loup, G., Serna, A., Iksal, S., George, S. 2016. Immersion and persistence: improving learners' engagement in authentic learning situations. Lecture Notes in Computer Science (Including Subseries Lecture Notes in Artificial Intelligence and Lecture Notes in Bioinformatics), 9891 LNCS, 410-415. https://doi.org/10.1007/978-3-31945153-4_35

Luigini, A., Basso, A. 2021. Heritage education for primary age through an immersive serious game. In Springer Tracts in Civil Engineering (157-174). Springer. https://doi.org/10.1007/978-3-030-49278-6_10

Lv, C., Xing, Y., Zhang, J., Na, X., Li, Y., Liu, T., Cao, D., Wang, F.Y. 2018. Levenberg-marquardt backpropagation training of multilayer neural networks for state estimation of a safety-critical cyber-physical system. IEEE Transactions on Industrial Informatics, 14, 3436-3446. https://doi.org/10.1109/TII.2017.2777460

Ma, M., Oikonomou, A.V., Jain, L.C. 2011. Serious Games and edutainment applications. Springer-Verlag London Ltd.

Maran, N.J., Glavin, R.J. 2003. Low-to high-fidelity simulation - a continuum of medical education? Medical Education, 37, 22-28. https://doi.org/10.1046/j.13652923.37.s1.9.x

Mentzelopoulos, M., Ferguson, J., Protopsaltis, A. 2015. Perceptually captured gesture interaction with immersive information retrieval environments: An experimental 
framework for testing and rapid iteration. Proceedings of 2015 International Conference on Interactive Mobile Communication Technologies and Learning, IMCL 2015, 307-311. https://doi.org/10.1109/IMCTL.2015.7359608

Mentzelopoulos, M., Parrish, J., Kathrani, P., Economou, D. 2016. REVRLaw: An immersive way for teaching criminal law using virtual reality. Communications in Computer and Information Science, 621, 73-84. https://doi.org/10.1007/978-3-319-41769-1_6

Michael, D. 2006. Serious Games: games that educate, train and inform /. Thomson Course Technology.

Misfeldt, M., Gjedde, L. 2015. What is a game for geometry teaching: Creative, embodied and immersive aspects. Proceedings of the European Conference on GamesBased Learning, 378-386.

Moore, 2007. United states patent - Microsoft Sidewinder.

Nima, U., Wangdi, R., Hauge, J.B. 2019. A serious game for competence development in internet of things and knowledge sharing. IEEE International Conference on Industrial Engineering and Engineering Management, 1786-1790. https://doi.org/10.1109/IEEM.2018. 8607737

O’Connor, S., Colreavy-Donnelly, S., Dunwell, I. 2020. Fostering engagement with cultural heritage through immersive vr and gamification. In Springer Series on Cultural Computing (301-321). Springer. https://doi.org/10.1007/978-3-030-37191-3_16

PC Mag 1984-10-02, 1984. Microsoft flight simulator 2.0. http://archive.org/details/PC-Mag-1984-10-02

PC Word, 1984. Microsoft flight simulator 2.0. http://archive.org/details/pcworld512unse

Peng, S.-T., Hsu, S.-Y., Hsieh, C.-K. 2015. An interactive immersive serious game application for kunyu quantu world map. ISPRS Annals of Photogrammetry, Remote Sensing and Spatial Information Sciences, II-5(W3), 221-227. https://doi.org/10.5194/isprsannals-II-5-W3221-2015

Perez-Gutierrez, B., Uribe-Quevedo, A., Vega-Medina, L., Salgado, J.S., Jaimes, N., Perez, O. 2020. Immersive and Non-Immersive VR Percutaneous Coronary Intervention Simulation for Acute Myocardial Infarction. 2020 IEEE 8th International Conference on Serious Games and Applications for Health, SeGAH 2020. https://doi.org/10.1109/SeGAH49190.2020.9201902

Pivec, M., Kearney, P. 2007. Games for learning and learning from games. Informatica, 31, 419-423.

Prensky, M. 2007. Digital game-based learning. In M. St. Paul (Ed.), Computers in Entertainment (2nd edition, 1). Paragon House. https://doi.org/10.1145/950566.950596

Rahouti, A., Lovreglio, R., Datoussaïd, S., Descamps, T. 2021. Prototyping and validating a non-immersive virtual reality serious game for healthcare fire safety training. Fire Technology. https://doi.org/10.1007/s10694-02101098-x

Rankin, Y.A., Mcneal, M., Shute, M.W., Gooch, B. 2008. User centered game design: Evaluating massive multiplayer online role playing games for second language acquisition. Sandbox Symposium 2008, 43-49. https://91-592-722.wiki.uml.edu/file/view/mmo_games social networking.pdf

Ratan, R., Ritterfeld, U. 2009. Classifying Serious Games. In Ritterfeld, U., Cody, M., Vorderer, P. (eds.) Serious Games: Mechanisms and effects.

Rooney, P. 2012. A theoretical framework for serious game design: Exploring pedagogy, play and fidelity and their implications for the design process. International Journal of Game-Based Learning, 2, 41-60. https://doi.org/10.4018/ijgbl.2012100103

Sanford, K., Starr, L.J., Merkel, L., Kurki, S.B. 2015. Serious Games: video games for good? E-Learning and Digital Media, 12, 90-106. https://doi.org/10.1177/2042753014558380

Sanmugan, Ma. A., Mohamed, H., Abdullah, Z.B. 2014. Gamification and Serious Games: -The enigma and the use in education. ISQAE 2014, 3rd International Seminar on Quality and Affordable Education 2014.

Saurina, N., Purwaningrum, S.W., Kukuh, A. 2016. Bikers game-Simulation game to introduce calories for children in aged 7-8 years old. International Journal of Emerging Trends \& Technology in Computer Science, 5, 67-74.

Sawyer, B., Smith, P. 2008. Serious Games taxonomy. Serious Games Summit.

Sax, H.S., Longtin, Y. 2011. Immersive hand hygiene trainer for physicians-a story-based serious game. 29. https://doi.org/10.1186/1753-6561-5-S6-O31

Shen, C., Wang, H., Ritterfe, U. 2009. Serious Games and seriously fun games can they be one and the same? http://nobelprize.org/

Simulator, M.F. 2019. Feature discovery series episode 2: Weather. Microsoft Flight Simulator. https://www.youtube.com/watch?v=i8TGT87Fxyw

Simulator, M.F. 2020. Feature discovery series episode 8: IFR. Microsoft Flight Simulator. https://www.youtube.com/watch? v=L8_UZT8nL-U

Sinclair, J.-L. 2020. The role of audio in interactive and immersive environments. In Principles of Game Audio and Sound Design (7-23). Focal Press. https://doi.org/10.4324/9781315184432-2

Smith, H.C. 2001. Teaching flight test engineering with a PC-based simulator. ASEE Annual Conference Proceedings, 9477-9483. https://doi.org/10.18260/1-29865

Valladares-Rodríguez, S., Pérez-Rodríguez, R., AnidoRifón, L., Fernández-Iglesias, M. 2016. Trends on the application of Serious Games to neuropsychological evaluation: A scoping review. In Journal of Biomedical Informatics, 64, 296-319. Academic Press Inc. https://doi.org/10.1016/j.jbi.2016.10.019

Vlachopoulos, D., Makri, A. 2017. The effect of games and simulations on higher education: a systematic literature review. International Journal of Educational Technology in Higher Education, 22. https://doi.org/10.1186/s41239017-0062-1 


\section{International Journal of Applied Science and Engineering}

Adisusilo et al., International Journal of Applied Science and Engineering, 18(5), 2021229

Wang, C., Ma, Q., Zhu, D., Chen, H., Yang, Z. 2013. Realtime control of $3 \mathrm{D}$ virtual human motion using a depthsensing camera for agricultural machinery training. Mathematical and Computer Modelling, 58, 782-789. https://doi.org/10.1016/j.mcm.2012.12.026

Wang, S.T., Liu, L.M., Wang, S.M. 2018. The design and evaluate of virtual reality immersive learning - The case of serious game "Calcium Looping for Carbon Capture." 2018 International Conference on System Science and Engineering, ICSSE 2018. https://doi.org/10.1109/ICSSE.2018.8520002

Yang, M.C., Wang, Z.W., Zhang, Y.K., Li, K.Y. 2019. Improving immersion in the serious education games: A new designing framework. Proceedings - 2019 8th International Congress on Advanced Applied Informatics, IIAI-AAI 2019, 296-301. https://doi.org/10.1109/IIAIAAI.2019.00066

Zyda, M. 2005. From visual simulation to virtual reality to games. Computer, 38, 25-32. https://doi.org/10.1109/MC.2005.297 